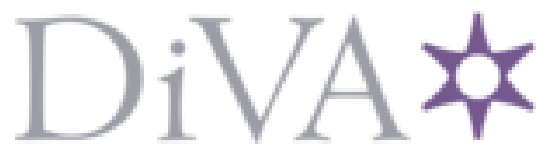

http://www.diva-portal.org

This is the published version of a paper presented at International conference of numerical analysis and applied mathematics (ICNAAM 2017), Thessaloniki, Greece, September 25-30, 2017.

Citation for the original published paper:

Cohen, D. (2018)

Numerical discretisations of stochastic wave equations

In: International conference of numerical analysis and applied mathematics (ICNAAM 2017), 1 (pp. 1-5). American Institute of Physics (AIP)

AIP Conference Proceedings

https://doi.org/10.1063/1.5043646

N.B. When citing this work, cite the original published paper.

Permanent link to this version:

http://urn.kb.se/resolve?urn=urn:nbn:se:umu:diva-150238 


\section{Numerical discretisations of stochastic wave equations}

Cite as: AIP Conference Proceedings 1978, 020001 (2018); https://doi.org/10.1063/1.5043646 Published Online: 10 July 2018

\section{David Cohen}

\section{ARTICLES YOU MAY BE INTERESTED IN}

Conference Details: International Conference of Numerical Analysis and Applied Mathematics (ICNAAM 2017)

AIP Conference Proceedings 1978, 010002 (2018); https://doi.org/10.1063/1.5043644

Preface: International Conference of Numerical Analysis and Applied Mathematics (ICNAAM 2017)

AIP Conference Proceedings 1978, 010001 (2018); https://doi.org/10.1063/1.5043643

The numerical solution of random ODES

AIP Conference Proceedings 1978, 020002 (2018); https://doi.org/10.1063/1.5043647

Lock-in Amplifiers Find out more today

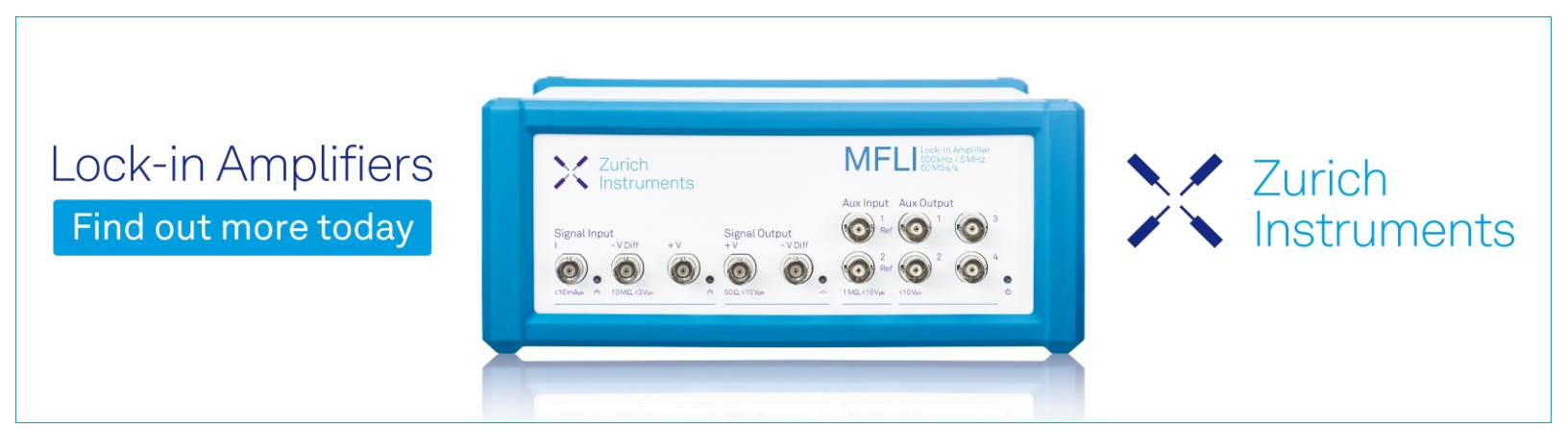




\title{
Numerical Discretisations of Stochastic Wave Equations
}

\author{
David Cohen ${ }^{1, a)}$ \\ ${ }^{1}$ Department of Mathematics and Mathematical Statistics, Umeå University, SE-90187 Umeå \\ ${ }^{a}$ Corresponding author: david.cohen@umu.se \\ URL: http://snovit.math.umu.se/ david/
}

\begin{abstract}
This extended abstract starts with a brief introduction to stochastic partial differential equations with a particular focus on stochastic wave equations. Various numerical experiments for this stochastic partial differential equation are presented. Finally, we point out results from the literature on the numerical analysis of stochastic wave equations.

Keywords: Stochastic partial differential equations. Stochastic wave equations. Numerical methods. Convergence. Long-time behaviour.

PACS: $02.50 . E y$, 02.60.x, 02.60.Cb, 02.70.c
\end{abstract}

\section{INTRODUCTION}

The last decades have seen an increase of studies of extensions of partial differential equations (PDEs) to stochastic partial differential equations (SPDEs). These extensions have become more and more important in various fields of applications from natural sciences, engineering, or financial mathematics, where various type of uncertainties need to be modelled $[1,2,3,4,5]$. Closed-form solutions to most of these SPDEs are rarely known and thus numerical simulations and a deep analysis of numerical schemes are mandatory.

In the talk and in the present extended abstract, we will first give a very concise introduction to stochastic partial differential equations with a particular focus on the stochastic wave equation (Section 2). A brief presentation of general numerical techniques for SPDEs is then given in Section 3. We conclude the extended abstract with various numerical experiments as well as a short review on theoretical results from the literature on the numerical analysis of stochastic wave equations (see Section 4).

\section{STOCHASTIC WAVE EQUATIONS}

Imagine a strand of DNA molecule floating in a fluid: the fluid's molecules are constantly hitting the DNA molecule (which could be modelled as a long elastic string) at random points in time and space giving raise to a random force acting on the strand of DNA. This problem can be described by a complicated system of three stochastic wave equations [2, Section 1]. Further applications which could be described by stochastic wave equations include: the dilatation of shock waves throughout the sun from [2, Section 1], as well as the motion of randomly forced strings [6, 7].

To fix notations, a semi-linear stochastic wave equation with multiplicative noise in a smooth domain $\mathcal{D} \subset \mathbb{R}^{d}, d=1,2,3$, can be written as

$$
\begin{array}{ll}
\mathrm{d} \dot{u}-\Delta u \mathrm{~d} t=f(u) \mathrm{d} t+g(u) \mathrm{d} W & \text { in } \mathcal{D} \times(0, \infty), \\
u=0 & \text { in } \partial \mathcal{D} \times(0, \infty), \\
u(\cdot, 0)=u_{0}, \dot{u}(\cdot, 0)=v_{0} & \text { in } \mathcal{D},
\end{array}
$$

where the unknown solution (a stochastic process) reads $u=u(x, t)$. Here, the "." denotes the time derivative $\frac{\partial}{\partial t}$. The Laplacian in $\mathbb{R}^{d}$ is denoted by $\Delta$. The nonlinearities $f$ and $g$ are given functions. The stochastic process (the noise) $\{W(t)\}_{t \geq 0}$ is an $L_{2}(\mathcal{D})$-valued (possibly cylindrical) $Q$-Wiener process with a given covariance operator $Q$. The given initial data $u_{0}$ and $v_{0}$ could be random variables. We remark that the term $g(u) \mathrm{d} W$ (in the special case of additive noise, i. e. when $g(u)=1$ ) in the above equation could model the random force acting on the DNA example from above.

A precise mathematical formulation of SPDEs needs tools from functional analysis, theory of PDEs, probability theory, and stochastic analysis. This is out of the scope of the present extended abstract and we refer the reader to, e.g., the monographs $[3,8,9]$ as well as the excellent lecture notes $[10,11,12,13,14,15]$.

International Conference of Numerical Analysis and Applied Mathematics (ICNAAM 2017)

AIP Conf. Proc. 1978, 020001-1-020001-5; https://doi.org/10.1063/1.5043646

Published by AIP Publishing. 978-0-7354-1690-1/\$30.00 


\section{NUMERICAL DISCRETISATIONS}

In order to numerically approximate solutions to SPDEs, such as equation (1), one must:

- $\quad$ discretise the infinite dimensional Hilbert space, where the solution lives, leading to a spatial discretisation of the SPDE;

- $\quad$ discretise the infinite dimensional Hilbert space, where the noise lives, leading to an approximation of the noise;

- $\quad$ discretise the time interval, where one wants to solve the equation, leading to a temporal discretisation of the SPDE.

Let us mention that various type of convergence for the numerical solutions of SPDEs exist e.g.:

- $\quad$ strong convergence (i. e. the pathwise error averaged over all paths);

- weak convergence (or approximation of expectations of functionals of the solutions);

- almost-sure convergence;

- convergence in probability.

Observe that this may require numerical approximations of mathematical expectations, using for instance Monte-Carlo-type techniques.

In the recent years, various computational techniques have been constructed and analysed to deal with the above issues. The interested reader is referred to, for instance, the works $[16,17,18,8]$ for extended details and further references.

\section{NUMERICAL EXPERIMENTS AND THEORETICAL RESULTS}

This section presents three different types of numerical experiments on the stochastic wave equation (1) and provides a concise review of related results from the literature. For ease of presentation, we only consider the one-dimensional linear case with additive noise (written as a first order system on the right)

$$
\begin{array}{llll}
\mathrm{d} \dot{u}-\Delta u \mathrm{~d} t=\mathrm{d} W & \text { in }(0,1) \times(0, \infty), & \mathrm{d}\left[\begin{array}{l}
u \\
\dot{u}
\end{array}\right]=\left[\begin{array}{ll}
0 & I \\
\Delta & 0
\end{array}\right]\left[\begin{array}{l}
u \\
\dot{u}
\end{array}\right] \mathrm{d} t+\left[\begin{array}{l}
0 \\
I
\end{array}\right] \mathrm{d} W(t), \\
u(0, t)=u(1, t)=0 & \text { for } t>0, & \text { or } & {\left[\begin{array}{c}
u(x, 0) \\
\dot{u}(x, 0)
\end{array}\right]=\left[\begin{array}{c}
\cos (\pi(x-1 / 2)) \\
0
\end{array}\right],} \\
u(x, 0)=\cos (\pi(x-1 / 2)), \dot{u}(x, 0)=0 & \text { for } x \in(0,1), &
\end{array}
$$

with a noise having covariance operator $Q=(-\Delta)^{-1 / 2}$.

Expected value of the energy. In the deterministic setting, the linear wave equation (that is equation (2) without noise) is a Hamiltonian PDE, wherein the total energy (or Hamiltonian) of the problem is conserved for all times. In the above stochastic case, it can be shown that the expected value of the energy along the exact solution grows linearly with time [19,20,21]

$$
\mathbb{E}[\underbrace{\frac{1}{2} \int_{\mathcal{D}}\left(|\dot{u}(t)|^{2}+|\nabla u(t)|^{2}\right) \mathrm{d} x}_{\text {energy at time } t}]=\mathbb{E}[\underbrace{\frac{1}{2} \int_{\mathcal{D}}\left(|\dot{u}(0)|^{2}+|\nabla u(0)|^{2}\right) \mathrm{d} x}_{\text {initial energy }}]+\frac{t}{2} \operatorname{Tr}(Q), \quad t \geq 0 .
$$

What about the behaviour of numerical solutions?

To illustrate the behaviour of numerical solutions to the linear stochastic wave equation (2), we first discretise the problem with a standard finite element method (FEM) in space (with mesh $h=0.1$ ) and then compute the expected value of the energy for the following time integrators (with large step-size $k=0.1$ ):

- $\quad$ the explicit stochastic trigonometric method from [20]

$$
\left[\begin{array}{l}
u_{n+1} \\
\dot{u}_{n+1}
\end{array}\right]=\left[\begin{array}{cc}
\cos \left(k(-\Delta)^{1 / 2}\right) & (-\Delta)^{-1 / 2} \sin \left(k(-\Delta)^{1 / 2}\right) \\
-(-\Delta)^{1 / 2} \sin \left(k(-\Delta)^{1 / 2}\right) & \cos \left(k(-\Delta)^{1 / 2}\right)
\end{array}\right]\left[\begin{array}{l}
u_{n} \\
\dot{u}_{n}
\end{array}\right]+\left[\begin{array}{c}
(-\Delta)^{-1 / 2} \sin \left(k(-\Delta)^{1 / 2}\right) \\
\cos \left(k(-\Delta)^{1 / 2}\right)
\end{array}\right] \Delta W^{n},
$$

where $\Delta W^{n}=W\left(t_{n+1}\right)-W\left(t_{n}\right)$ denote the Wiener increments, and $u_{n} \approx u(n k)$;

- the backward Euler-Maruyama scheme, see for example [22],

$$
\left[\begin{array}{l}
u_{n+1} \\
\dot{u}_{n+1}
\end{array}\right]=\left[\begin{array}{l}
u_{n} \\
\dot{u}_{n}
\end{array}\right]+k\left[\begin{array}{ll}
0 & I \\
\Delta & 0
\end{array}\right]\left[\begin{array}{l}
u_{n+1} \\
\dot{u}_{n+1}
\end{array}\right]+\left[\begin{array}{l}
0 \\
I
\end{array}\right] \Delta W^{n}
$$

- $\quad$ the Crank-Nicolson-Maruyama scheme, see for example [23],

$$
\left[\begin{array}{c}
u_{n+1} \\
\dot{u}_{n+1}
\end{array}\right]=\left[\begin{array}{l}
u_{n} \\
\dot{u}_{n}
\end{array}\right]+\frac{k}{2}\left[\begin{array}{cc}
0 & I \\
\Delta & 0
\end{array}\right]\left(\left[\begin{array}{c}
u_{n+1} \\
\dot{u}_{n+1}
\end{array}\right]+\left[\begin{array}{l}
u_{n} \\
\dot{u}_{n}
\end{array}\right]\right)+\left[\begin{array}{l}
0 \\
I
\end{array}\right] \Delta W^{n}
$$




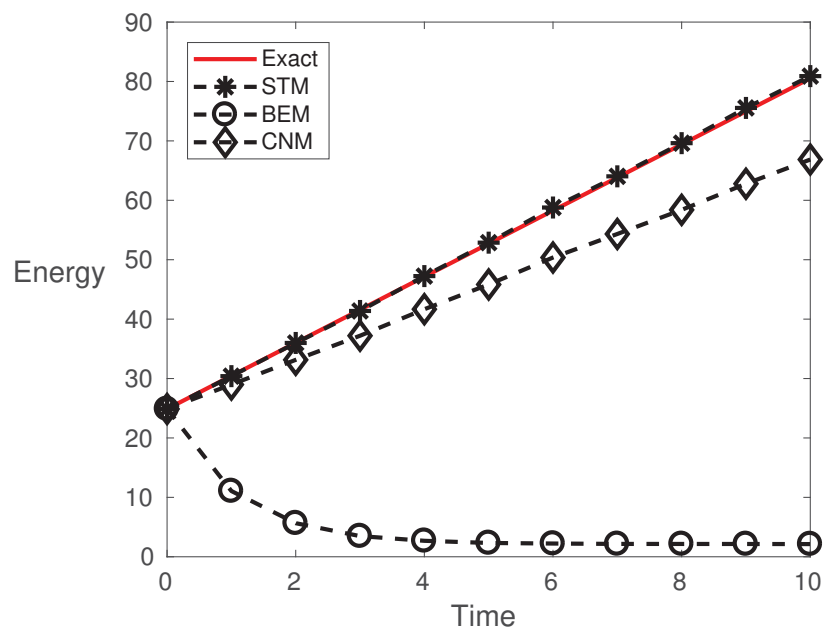

FIGURE 1. Expected value of the energy on the time interval $[0,10]$ for the stochastic trigonometric method (STM), the backward Euler-Maruyama scheme (BEM) and the Crank-Nicolson-Maruyama scheme (CNM). The expected value of the exact solution is displayed in solid red line. All expected values are approximated using $M_{S}=5000$ samples.

The results are displayed in Fig. 1, where one can observe the excellent long-time properties of the stochastic trigonometric method with respect to the conservation of the drift in the expected value of the energy.

The proof of the exact preservation of the expected value of the energy by the stochastic trigonometric methods is given in [20]. Furthermore, the behaviour of this numerical solution for nonlinear problems is studied in [21]. Observe that preliminary results on numerical discretisations (by a Fourier pseudo-spectral method and midpoint-type schemes) of one-dimensional nonlinear stochastic wave equations with additive noise are presented in [24].

Strong convergence. We compute the root mean-square errors

$$
\sqrt{\mathbb{E}\left[\left\|u(T)-u_{N}\right\|_{L_{2}(\mathcal{D})}^{2}\right]}
$$

where $u_{N}$ are numerical approximations of the exact solution $u(T)$ at time $T=0.25$ given by the above mentioned time integrators for time step ranging from $k=2^{-3}$ to $2^{-10}$ and a fixed FEM mesh of size $h=2^{-8}$. The reference solution is computed with the stochastic trigonometric method with $k_{\text {ref }}=2^{-20}$ and $h_{\text {ref }}=2^{-8}$. Figure 2 (left) presents a loglog plot of these errors, where one can observe the orders of convergence of these time integrators.

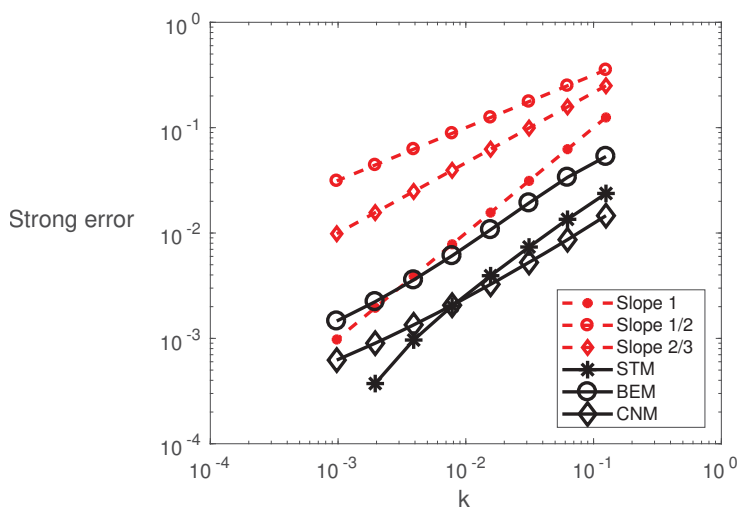

(a) Strong errors. All expected values are approximated using $M_{s}=2500$ samples.

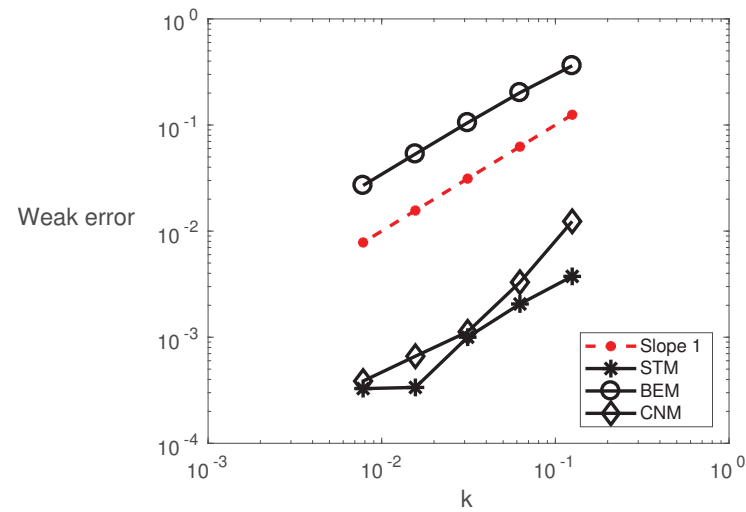

(b) Weak errors. All expected values are approximated using $M_{s}=$ 1000000 samples.

FIGURE 2. Strong (left) and weak (right) errors of the stochastic trigonometric method (STM), the backward EulerMaruyama scheme (BEM) and the Crank-Nicolson-Maruyama scheme (CNM). 
The topic of strong convergence has been extensively studied in the literature. Rates of convergence for spatial discretisations can be found in [25, 21] for FEM, in [26, 27] for finite difference schemes, and in [28, 19, 29] for spectral-type schemes. Results on time integrations for stochastic wave equations are found in [30] for $I$-stable rational approximations (e.g. linear-implicit EulerMaruyama scheme), in [26] for the leap-frog scheme, and in [20, 29, 31, 21, 32] for stochastic trigonometric/exponential-type integrators.

Weak convergence. We finally illustrate the weak errors of these time integrators by measuring the errors

$$
\left|\mathbb{E}[\sin (u(T))]-\mathbb{E}\left[\sin \left(u_{N}\right)\right]\right|,
$$

where $u_{N}$ are numerical approximations of the exact solution $u(T)$ at time $T=0.125$. We use a reference solution computed with the stochastic trigonometric method using $k_{\text {ref }}=2^{-15}$ and $h_{\text {ref }}=2^{-8}$. The numerical results are presented in Fig. 2 (right).

Theoretical results on weak approximation of stochastic wave equations can be found in [33, 30] (FEM and $I$-stable rational approximations), in [34] (leap-frog scheme), in [31] (stochastic trigonometric/exponential-type integrators), and very recently in $[35,36]$ (spectral Galerkin approximations in space).

\section{ACKNOWLEDGMENTS}

The author would like to thank A. Jentzen, A. Lang, and X. Wang for interesting discussions. This work was partially supported by the Swedish Research Council (VR) (project nr. 2013 - 4562). The computations were performed on resources provided by the Swedish National Infrastructure for Computing (SNIC) at HPC2N, Umeå University.

\section{REFERENCES}

[1] C. Prévôt and M. Röckner, A Concise Course on Stochastic Partial Differential Equations, Lecture Notes in Mathematics, Vol. 1905 (Springer, Berlin, 2007), pp. vi+144.

[2] R. Dalang, D. Khoshnevisan, C. Mueller, D. Nualart, and Y. Xiao, A Minicourse on Stochastic Partial Differential Equations, Lecture Notes in Mathematics, Vol. 1962 (Springer-Verlag, Berlin, 2009), pp. xii+216, held at the University of Utah, Salt Lake City, UT, May 8-19, 2006, Edited by Khoshnevisan and Firas Rassoul-Agha.

[3] G. Da Prato and J. Zabczyk, Stochastic Equations in Infinite Dimensions, 2nd ed., Encyclopedia of Mathematics and its Applications, Vol. 152 (Cambridge University Press, Cambridge, 2014), pp. xviii+493.

[4] A. Lang, in Computer Vision: A Reference Guide, edited by K. Ikeuchi (Springer, 2014), pp. 770-775.

[5] W. Liu and M. Röckner, Stochastic Partial Differential Equations: an Introduction, Universitext (Springer, Cham, 2015), pp. vi+266.

[6] E. Cabaña, Z. Wahrscheinlichkeitstheorie und Verw. Gebiete 15, 111-130 (1970).

[7] E. Orsingher, Ann. Inst. H. Poincaré Sect. B (N.S.) 18, 367-394 (1982).

[8] G. J. Lord, C. E. Powell, and T. Shardlow, An Introduction to Computational Stochastic PDEs, Cambridge Texts in Applied Mathematics (Cambridge University Press, New York, 2014), pp. xii+503.

[9] S. V. Lototsky and B. L. Rozovsky, Stochastic partial differential equations, Universitext (Springer, Cham, 2017), pp. xiv +508 .

[10] E. Pardoux, Stochastic partial differential equations, April 2007.

[11] J. van Neerven, Stochastic evolution equations, 2007.

[12] M. Hairer, An introduction to stochastic PDEs, July 2009.

[13] M. Kovács and S. Larsson, Introduction to stochastic partial differential equations, January 2009.

[14] C.-E. Bréhier, A short introduction to stochastic PDEs, March 2014.

[15] A. Jentzen, An introduction to stochastic partial differential equations, June 2017.

[16] A. Jentzen and P. E. Kloeden, Milan J. Math. 77, 205-244 (2009).

[17] A. Jentzen and P. E. Kloeden, Taylor Approximations for Stochastic Partial Differential Equations, CBMS-NSF Regional Conference Series in Applied Mathematics, Vol. 83 (Society for Industrial and Applied Mathematics (SIAM), Philadelphia, PA, 2011), pp. xiv+220.

[18] R. Kruse, Strong and Weak Approximation of Semilinear Stochastic Evolution Equations, Lecture Notes in Mathematics, Vol. 2093 (Springer, Cham, 2014), pp. xiv+177.

[19] H. Schurz, Discrete Contin. Dyn. Syst. Ser. S 1, 353-363 (2008).

[20] D. Cohen, S. Larsson, and M. Sigg, SIAM J. Numer. Anal. 51, 204-222 (2013).

[21] R. Anton, D. Cohen, S. Larsson, and X. Wang, SIAM J. Numer. Anal. 54, 1093-1119 (2016).

[22] P. E. Kloeden and E. Platen, Numerical solution of stochastic differential equations, Applications of Mathematics (New York), Vol. 23 (Springer-Verlag, Berlin, 1992), pp. xxxvi+632.

[23] E. Hausenblas, Potential Anal. 18, 141-186 (2003).

[24] H. Schurz, Dyn. Contin. Discrete Impuls. Syst. Ser. A Math. Anal. 14, 74-78 (2007).

[25] M. Kovács, S. Larsson, and F. Saedpanah, SIAM J. Numer. Anal. 48, 408-427 (2010). 
[26] J. B. Walsh, Illinois J. Math. 50, 991-1018 (2006).

[27] L. Quer-Sardanyons and M. Sanz-Solé, Potential Anal. 24, 303-332 (2006).

[28] Y. Cao and L. Yin, Commun. Pure Appl. Anal. 6, 607-617 (2007).

[29] X. Wang, S. Gan, and J. Tang, SIAM J. Sci. Comput. 36, A2611-A2632 (2014).

[30] M. Kovács, S. Larsson, and F. Lindgren, BIT Numerical Mathematics 53, 497-525 (2013).

[31] X. Wang, J. Sci. Comput. 64, 234-263 (2015).

[32] D. Cohen and L. Quer-Sardanyons, IMA J. Numer. Anal. 36, 400-420 (2016).

[33] M. Kovács, S. Larsson, and F. Lindgren, BIT 52, 85-108 (2012).

[34] E. Hausenblas, J. Comput. Appl. Math. 235, 33-58 (2010).

[35] L. Jacobe de Naurois, A. Jentzen, and T. Welti, ArXiv e-prints 1-27aug (2015), arXiv:1508.05168

[36] L. Jacobe de Naurois, A. Jentzen, and T. Welti, ArXiv e-prints 1-9jan (2017), arXiv:1701.04351. 